\title{
L'EXPRESSION DE LA PERCEPTION DANS LE LEXICON DE BUDA
} (1825)

\author{
Maria ALDEA \\ Université Babeş-Bolyai, Cluj, Roumanie
}

\begin{abstract}
En) : In our paper, we analyze the manner of expressing perception in an old Romanian dictionary published at the Typography of Buda in 1825, a lexicographical masterpiece created by the members of the Transylvanian School. Based on this dictionary, we would like to compile an inventory of the words of perception such as they are preserved, while highlighting the normative aspects with regard to the current standards, their semantic development and their lexicographical treatment.
\end{abstract}

Keywords (En) : the Lexicon of Buda; physical perception; sensory perception; Romanian verbs; word family; derivation

Mots-clés (Fr) : le Lexicon de Buda ; perception physique ; perception sensorielle ; verbes roumains ; famille de mots ; dérivation

\section{Introduction}

Personne ne pourrait contester aujourd'hui la complexité des rôles joués par les ouvrages lexicographiques. Outils linguistiques et culturels, ils conservent « la mémoire sémantique » d'une époque et apportent des informations précieuses à la fois sur la diffusion des mots, sur les normes linguistiques en vogue à une époque donnée et surtout sur la formation intellectuelle et les différents choix de leurs rédacteurs (ALDEA, 2007 ; ALDEA, 2012).

Dans notre étude, nous allons nous pencher sur les diverses manières d'exprimer la perception décelables dans un dictionnaire roumain ancien, connu dans la littérature roumaine de spécialité sous le nom du Lexicon de Buda (pour des recherches sur l'expression de la perception dans l'espace roumain, voir DIMA, 2002 et NICULA, 2012). D'une part, nous envisageons de dresser un inventaire de mots de la perception; pour leur sélection, nous nous servirons, dans un premier temps, des équivalents latins disponibles, et ensuite de l'analyse sémique, tout en nous rapportant aux dictionnaires roumains actuels. D'autre part, nous voudrions mettre en évidence à la fois certains aspects normatifs anciens par rapport aux normes actuelles, l'évolution sémantique des mots - dans le cas où elle est mentionnée, et leur traitement lexicographique. Dans cette étape, nous éviterons de prendre en considération d'autres approches, fussent-elles contrastives ou ethnolinguistiques. $\mathrm{Au}$ final, nous aurons une perspective plus élargie sur la circulation et l'évolution en diachronie de ces mots de la perception dans une aire géographique où la langue roumaine ne bénéficiait pas encore de reconnaissance officielle. Mais, avant de passer à l'analyse proprement dite, il convient de présenter brièvement ce dictionnaire extraordinaire.

La rédaction du Lexicon de Buda (désormais abrégé : $L B^{e}$ ) s'est étendue sur plus de trente ans, étant assurée par plusieurs savants de l'École latiniste de Transylvanie. Cette école s'affirme comme un mouvement idéologique et culturel censé faciliter l'émancipation des Roumains et « éveiller leur conscience » nationale et sociale (cf. MUNTEANU, 1997: 6; notre traduction). Il faut mentionner qu'à l'époque, la 
population roumaine de la Transylvanie ne jouissait ni de droits ni de privilèges comme les autres ethnies présentes sur son territoire (les Hongrois, les Saxons et les Sicules), n'ayant que le simple statut de population "tolérée ». C'est grâce aux efforts intellectuels de ces grands érudits transylvains, parmi lesquels Samuil Micu, Vasile Coloşi, Ioan Corneli, Petru Maior, Ioan Theodorovici, Alexandru Teodori et tant d'autres, qu'un dictionnaire en quatre langues (à savoir le roumain, le latin, le hongrois et l'allemand) paraîtra en 1825 à l'Imprimerie Royale de l'Université de Buda. "Toutes proportions historiques gardées », ces savants « ont fait pour la culture roumaine [...] ce que Montesquieu, Diderot et Voltaire ont accompli pour la culture française et Lessing pour l'allemande » (cf. MUNTEANU, 1997 : 6; notre traduction).

Ce dictionnaire réalisé par un groupe d'érudits transylvains représente un moment charnière pour la lexicographie roumaine moderne. C'est le premier grand dictionnaire étymologique et explicatif imprimé (ALDEA, 2013). Grâce à sa parution dans l'espace plurilingue et pluriethnique de la Transylvanie, la langue roumaine affirmait enfin son origine latine - en un mot, ses lettres de noblesse.

\section{Exprimer la perception dans le Lexicon de Buda}

L'examen des entrées du $L B^{e}$ nous a permis d'observer qu'un large nombre de mots renvoyait en effet au champ de la perception. Ces mots appartiennent à des classes grammaticales très différentes : nous pouvons ainsi y trouver des noms ou des adjectifs, tout comme des adverbes ou des verbes. Vu l'ampleur de l'inventaire qu'on pourrait en construire à partir des données recensées, nous avons choisi de restreindre le champ de la présente étude à la seule classe des verbes, tout en nous focalisant sur les verbes qui décrivent une perception physique ou sensorielle. Il convient de souligner que nous avons choisi d'enregistrer dans l'Annexe non seulement des verbes de perception, mais aussi les dérivés de ces verbes sentiendi (des noms, des adjectifs et des adverbes) pour permettre aux spécialistes de se forger une image sur la productivité des bases verbales en question, et ce, même si ces dérivés ne constituent pas l'objectif proprement dit de notre analyse. De plus, nous allons mentionner également plusieurs formes verbales qui ne présentaient pas, à l'époque, de sens sensoriel et ne renvoyaient pas encore à la perception (voir infra 2.4 ; cf. $L B^{e}$, s.v. Desmerdu, Jingảşescu, Mảngảiu).

Le traitement lexicographique consacré à ces vocables de la perception physique n'est pourtant ni uniforme ni cohérent. Ainsi, nous pouvons nous rapporter, par exemple, à la manière de rendre en roumain la définition des mots. Il $\mathrm{y}$ a certains verbes dont la définition roumaine est donnée soit à travers un commentaire linguistique, soit par des équivalents synonymiques; dans certains cas, on n'en a que les correspondants en latin, en hongrois ou en allemand, voire de simples renvois au mot vedette. Malgré cette lacune, nous avons pu réaliser la sélection des mots grâce aux équivalents latins.

Le prototype de cette classe des verbes de la perception est a simţi 'percevoir par les sens, sentir'.

Le $L B^{e}$ enregistre plusieurs formes de ce terme qui sont en fait des doublets lexicaux (cf. $L B^{e}$, s.v. Simţescu, Sẻnţescu, Simţiu, Sẻmţiu, Sẻnţiu) et phonétiques : 
i/ẻ (cf. $L B^{e}$, s.v. Simţescu, Sẻnţescu), i/ẻ (cf. LB e s.v. Simţiu, Sẻnţiu, Sẻmţiu), t/t (cf. $L B^{e}$, s.v. Simţiu, Sénţiu). En ce qui concerne le traitement lexicographique deces formes, on note que certaines ont leur catégorie grammaticale marquée (verbes actifs) (cf. $L B^{e}$, s.v. Simţescu, Sẻnţescu, Simţiu, Sẻnţiu), tandis que pour d'autres celle-ci est absente (cf. $L B^{e}$, s.v. Sẻmţiu). Si les titres des entrées simţiu, sâmţiu, sânţiu (cf. $L B^{e}$, s.v. Simţiu, Sẻmţiu, Sẻnţiu) sont définis par des renvois, pour les entrées simţesc, sânţesc (cf. $L B^{e}$, s.v. Simţescu, Sẻnţescu), les rédacteurs mentionnent deux sens; or, si le premier sens rend le sens de base en étant défini par les correspondants latins, hongrois et allemands, le second est exprimé à travers une expression idiomatique, à savoir a prinde de veste 'apprendre qqc., connaître par une information', accompagnée par des correspondants dans les trois autres langues du dictionnaire. De plus, ces deux dernières entrées bénéficient toutes les deux d'une notice étymologique, qui indique l'origine latine du mot, accompagnée de son équivalent en italien dans le cas du dernier exemple. Par rapport à la norme linguistique actuelle (i.e. simt 'je sens', simţi 'sentir', simţit 'senti'), on constate que les formes enregistrées par le $L B^{e}$ ne sont conservées et ne se retrouvent que dans les parlers roumains. De plus, l'infinitif long (rendu dans sa conjugaison complète, ce qui représentait encore la norme à l'époque - tout comme dans le cas des verbes dans les dictionnaires latins) est remplacé de nos jours par l'infinitif court (l'infinitif long fonctionne alors comme un nom).

Ajoutons également le fait que ce verbe constitue la base de formation d'autres mots, en particulier des noms : simţire 'sens' (cf. $L B^{e}$, s.v. Sẻnţire, Simţire), simţitoriu 'celui qui sent' (cf. $L B^{e}$, s.v. Sẻnţitoriu), nesimţire 'insensibilité ; défaillance, évanouissement' (cf. $L B^{e}$, s.v. Nesémţire, Nesimţire) ou des adjectifs : simţitoriu 'sensible' (cf. $L B^{e}$, s.v. Simţitoriu), nesimţitoriu 'insensible' (cf. $L B^{e}$, s.v. Nesémţitoriu). Ces nouveaux mots sont formés à l'aide de la dérivation par suffixes (où -re est le suffixe qui transforme le verbe à l'infinitif en un nom et -oriu est le suffixe du nom) et par préfixes (ne- est un préfixe négatif).

Comme équivalent synonymique de ce verbe prototypique, nous avons identifié le verbe a pricepe 'comprendre, saisir, entendre' (cf. LB', s.v. Precepu, Pricepu). Dans le cas de ce verbe aussi, on retrouve un doublet phonétique e/i ( $\mathrm{cf} . L B^{e}$, s.v. Precepu, Pricepu), qui renvoie surtout à un sens cognitif. Si, de nos jours, la forme littéraire actuelle est a pricepe (cf. $L B^{e}$, s.v. Pricepu), l'autre forme mentionnée dans le Lexicon (cf. $L B^{e}$, s.v. Precepu) existe seulement dans quelques parlers populaires.

Comme dans le cas du verbe a simţi 'percevoir par les sens, sentir', la dérivation par suffixes permet de créer des mots nouveaux : pricepere 'compréhension, intelligence, entendement' (cf. $L B^{e}$, s.v. Precépere), pricepătoriu 'savant' (cf. $L B^{e}$, s.v. Precepátoriu), preceput 'compétent, habile' (cf. $L B^{e}$, s.v. Preceputu), etc.

Nous avons pu identifier dans le $L B^{e}$ plusieurs verbes représentatifs pour chaque micro-champ lexical de la perception sensorielle. Dans ce qui suit, nous nous proposons de les examiner brièvement.

\section{Le micro-champ de la perception auditive}

Le micro-champ de la perception auditive est représenté par les verbes a auzi 'entendre' (cf. $L B^{e}$, s.v. Audiu), a asculta 'écouter' (cf. $L B^{e}$, s.v. Ascultu), a înțelege 
'comprendre' (cf. $L B^{e}$, s.v. Inţelegu), a oblici 'apprendre, entendre qqc. d'inattendu' (cf. $L B^{e}$, s.v. Oblicescu). Le sème qui organise ce micro-champ est [+ ouïe].

L'examen de ces quatre verbes nous a permis d'en saisir quelques aspects. Le prototype de la série est a auzi 'entendre' (cf. $L B^{e}$, s.v. Audiu), qui apparaît, dans la même entrée, sous trois formes graphiques pour exprimer l'indicatif présent à la $1^{\text {ère }}$ personne : audiu, aud, aud, dont la deuxième forme reproduit la variante littéraire, les deux autres formes étant plutôt spécifiques aux divers patois. Bien que le sens de base, celui par lequel on réalise l'expression de la perception, ne soit conservé qu'à la forme active (transitive) de ces verbes et qu'il ne soit exprimé que grâce à des équivalents latins, hongrois et allemands, le contexte d'emploi en roumain et dans les trois autres langues du dictionnaire apporte des informations très précises sur le sens.

Les verbes a auzi 'entendre', a asculta 'écouter' et a îțtelege 'comprendre' appartiennent au registre littéraire, tandis que la forme du verbe a oblici 'apprendre, entendre qqc. d'inattendu' est aujourd'hui vieillie ou obsolète. De plus, il convient de souligner que pour ce qui est du verbe a ințelege 'comprendre' (cf. $L B^{e}$, s.v. Inţelegu), il développe à la forme active deux valeurs ou connotations, à savoir une première connotation cognitive et une seconde connotation plutôt dénotative.

Ces quatre verbes sont tous à l'origine de la création de mots nouveaux qui sont, en règle générale, des noms et des adjectifs formés à l'aide des affixes lexicaux des suffixes ou des préfixes. Ainsi, pour a auzi 'entendre', nous avons la série : auzire 'ouïe' (cf. $L B^{e}$, s.v. Audire), auzitoriu ' audible, qui peut être entendu' (cf. $L B^{e}$, s.v. Auditoriu), neauzit 'inouï' (cf. $L B^{e}$, s.v. Ne audzitu); pour a asculta 'écouter', nous avons : ascultare 'obéissance' (cf. $L B^{e}$, s.v. Ascultare), ascultătoriu '1. celui qui écoute, 2. obéissant' (cf. $L B^{e}$, s.v. Ascultảtoriu), neascultare 'désobéissance' (cf. $L B^{e}$, s.v. Ne ascultare), neascultătoriu 'désobéissant' (cf. $L B^{e}$, s.v. Ne ascultảtoriu), pour a înțelege 'comprendre', nous disposons d'un large éventail de mots dérivés comme înţelegere 'compréhension, entendement' (cf. $L B^{e}$, s.v. Intelegere), înţelegătoriu 'compréhensif' (cf. $L B^{e}$, s.v. Inţelegetoriu), neînţelegere 'incompréhension' (cf. $L B^{e}$, s.v. Ne inţelégere), neînţelegătoriu 'incompréhensif' (cf. L $L B^{e}$, s.v. Ne inţeleghẻtoriu), neînţeles (nom) 'obscurité' (cf. $L B^{e}$, s.v. Ne inţelesu), neînţeles (adj.) 'incompris, confus' (cf. $L B^{e}$, s.v. Ne inţelesu), enfin, pour a oblici 'apprendre, entendre qqc. d'inattendu', nous disposons du nom oblicire 'entendement' (cf. $L B^{e}$, s.v. Oblicire).

\section{Le micro-champ de la perception gustative}

Ce micro-champ réunit les verbes a gusta 'goûter' (cf. LB', s.v. Gustu), a gustări 'goûter, déguster' (cf. L $L B^{e}$, s.v. Gustảrescu), a îmbuca 'goûter, manger un morceau' (cf. $L B^{e}$, s.v. Imbucu), a îmbucături 'goûter, manger un morceau' (cf. $L B^{e}$, s.v. Imbucáturescu). Le sème qui organise ce micro-champ de la perception gustative est [+ goût].

En examinant ces verbes, on a constaté que le prototype de la série était a gusta 'goûter' (cf. $L B^{e}$, s.v. Gustu). L'analyse de ce verbe nous permet d'observer qu'il est défini seulement par des contextes d'emploi accompagnés par des unités synonymiques latines, hongroises et allemandes. Bien délimité, le contexte 
d'emploi nous permet de mieux en saisir le sens. Le sens de base (et qui sert à exprimer la perception gustative) est conservé à la forme active (transitive) de ces verbes; on y ajoute la valeur neutre (ou le deuxième sens) du verbe a îmbucături 'goûter, manger un morceau' (cf. $L B^{e}$, s.v. Imbucảturescu). Les verbes a gusta 'goûter' (cf. $L B^{e}$, s.v. Gustu) et a îmbuca 'goûter, manger un morceau' (cf. $L B^{e}$, s.v. Imbиси) appartiennent au registre standard de la langue, tandis que les verbes a gustări 'goûter, déguster' (cf. $L B^{e}$, s.v. Gustảrescu) et a îmbucături 'goûter, manger un morceau' (cf. $L B^{e}$, s.v. Imbucảturescu) ne sont employés que très rarement et surtout au niveau régional.

On ajoutera également le fait que le nom gustu 'goût, saveur' (cf. $L B^{e}$, s.v. Gustu) sert à créer les mots suivants : a gusta 'goûter' (cf. LB', s.v. Gustu), gustos ou gustuos 'savoureux' (cf. LB', s.v. Gustosu), gustare 'goûter' (cf. LB' s.v. Gustare), negustat 'que l'on n'a pas goûté' (cf. LB', s.v. Negustatu); d'autres mots servant à exprimer la perception gustative sont formés à partir du mot bucă repris avec son sens de "joue » : a îmbuca 'goûter, manger un morceau' (cf. $L B^{e}$, s.v. Imbucu), imbucătură 'morceau' (cf. $L B^{e}$, s.v. Imbucảturả), imbucăturuţa 'un petit morceau' (cf. $L B^{e}$, s.v. Imbucáturuţa).

\section{Le micro-champ de la perception olfactive}

A mirosi 'sentir, percevoir par l'odorat' (cf. $L B^{e}$, s.v. Mirosu, Mirosescu), a amirosi 'sentir, percevoir par l'odorat' (cf. $L B^{e}$, s.v. Amirosu, Amirosescu), a odora 'odorer' (cf. $L B^{e}$, s.v. Odorezu), a ulma 'flairer' (cf. $L B^{e}$, s.v. Ulmu), a adurmeca 'flairer' (cf. $L B^{e}$, s.v. Adurmecu), a adulmeca 'flairer' (cf. $L B^{e}$, s.v. Adulmecu) sont des verbes appartenant au micro-champ de la perception olfactive, qui s'organise autour du sème [+ odorat].

Le prototype de la série est a mirosi 'sentir' (cf. $L B^{e}$, s.v. Mirosu, Mirosescu). L'examen de ce verbe nous permet de constater qu'il est enregistré sous deux mots vedettes distincts, i.e. mirosu et mirosescu, dont le second est défini à travers un renvoi au premier. En ce qui concerne la première forme, notons que sa définition est rendue par des contextes d'emploi rédigés en roumain et accompagnés par des unités lexicales ou des syntagmes synonymiques dans les trois autres langues du dictionnaire. Tous les verbes ont une valeur active (ou transitive) et leurs sens servent à exprimer la perception olfactive ; la seule exception est le verbe a amirosi 'sentir' (cf. $L B^{e}$, s.v. Amirosu), dont la valeur active est doublée par une valeur neutre. Trois formes verbales présentent des doublets lexicaux : i.e. a mirosi 'sentir, percevoir par l'odorat' (cf. LB', s.v. Mirosu, Mirosescu), a amirosi 'sentir, percevoir par l'odorat' (cf. $L B^{e}$, s.v. Amirosu, Amirosescu) et a adurmeca, a adulmeca 'flairer' (cf. $L B^{e}$, s.v. Adurmecu, Adulmecu). Le paradigme dont font partie les formes verbales a mirosi 'sentir' (cf. $L B^{e}$, s.v. Mirosu) et a adulmeca 'flairer' (cf. $L B^{e}$, s.v. Adulmecu) appartient au registre littéraire, tandis que les formes a mirosi 'sentir' (cf. $L B^{e}$, s.v. Mirosescu), a amirosi 'sentir' (cf. $L B^{e}$, s.v. Amirosu), a amirosi 'sentir' (cf. $L B^{e}$, s.v. Amirosescu) représentent des variantes populaires du verbe a mirosi ; la forme a adurmeca 'flairer' (cf. $L B^{e}$, s.v. Adurmecu) est une variante régionale du verbe a adulmeca (cf. $L B^{e}$, s.v. Adulmecu); le verbe a ulma 'flairer' (cf. $L B^{e}$, s.v. 
$U l m u$ ) est une forme régionale archaïque, tandis que le verbe a odora (cf. $L B^{e}$, s.v. Odorezu) est rarement employé.

Le nom miros 'odeur' (cf. $L B^{e}$, s.v. Mirosu) sert à créer des mots dérivés : a mirosi 'sentir' (cf. $L B^{e}$, s.v. Mirosu), miroseală 'odeur' (cf. $L B^{e}$, s.v. Mirosélả), mirosire 'odorat' (cf. $L B^{e}$, s.v. Mirosire), mirositoriu 'odorant' (cf. $L B^{e}$, s.v. Mirositoriu), mirositură 'odeur' (cf. $L B^{e}$, s.v. Mirositurả), nemirositoriu 'inodore' (cf. $L B^{e}$, s.v. Nemirositoriu); de même, le nom amiros 'odeur' (cf. $L B^{e}$, s.v. Amirosu) se trouve à la base des mots dérivés suivants : a amirosi 'sentir' (cf. $L B^{e}$, s.v. Amirosu), amiroseală 'odeur' (cf. $L B^{e}$, s.v. Amirosélả), amirosire 'odorat' (cf. $L B^{e}$, s.v. Amirosire), amirositoriu 'odorant' (cf. $L B^{e}$, s.v. Amirositoriu), amirositură 'odeur' (cf. $L B^{e}$, s.v. Amirositurả). Par contre, pour les verbes a ulma 'flairer' (cf. $L B^{e}$, s.v. Ulmu) et a odora 'odorer' (cf. $L B^{e}$, s.v. Odorezu), le $L B^{e}$ n'enregistre qu'un seul mot dérivé dans chaque cas: ulmare 'flairée' (cf. $L B^{e}$, s.v. Ulmare) et, respectivement, odoreală 'odeur' (cf. $L B^{e}$, s.v. Odorélả).

\section{Le micro-champ de la perception tactile}

Ce micro-champ est composé par les verbes a pipăi 'tâter, palper' (cf. $L B^{e}$, s.v. Pipảescu), a atinge 'toucher' (cf. $L B^{e}$, s.v. Atingu), a anina 'effleurer' (cf. $L B^{e}$, s.v. Aninu), a netezi 'caresser' (cf. LB', s.v. Netezescu), organisés autour du sème [+ toucher].

L'examen de ces verbes nous permet d'observer que tous sont des verbes actifs et que le prototype de la série est a pipăi 'palper, tâter, toucher' (cf. $L B^{e}$, s.v. Pipảescu). L'examen du verbe prototypique met en évidence le fait qu'on dispose d'une seule définition réalisée par l'intermédiaire des équivalents latins, hongrois et allemands, accompagnée d'une notice d'ordre étymologique. Le même type de définition se retrouve aussi bien dans la structure de l'article a atinge 'toucher' (cf. $L B^{e}$, s.v. Atingu) ; par contre, les verbes a anina 'effleurer' (cf. $L B^{e}$, s.v. Aninu) et a netezi 'caresser' (cf. $L B^{e}$, s.v. Netezescu) enregistrent deux sens, dont le second présente lui aussi le sème [+ toucher], accompagné dans le cas du verbe a anina par le sème [+ intensité] (légèrement), tandis que dans le cas du verbe a netezi, le complément désigne un animal. A l'exception des sens secondaires présentés par les deux derniers verbes qui ne sont plus valables dans la langue actuelle, tous les verbes font partie du registre littéraire de la langue.

Il convient de remarquer que, par rapport à d'autres séries de verbes de la perception, la capacité de dérivation est assez réduite : le verbe a pipăi 'tâter, palper' est à l'origine du nom pipăire 'toucher; attouchement' (cf. LB ${ }^{e}$, s.v. Pipảire) et de l'adjectif nepipăit 'impalpable' (cf. $L B^{e}$, s.v. Nepipáitu) ; le verbe a atinge 'toucher' vient avec ses dérivés : atingere 'toucher' (cf. $L B^{e}$, s.v. Atingere) et atins 'touché' (cf. $L B^{e}$, s.v. Atinsu) ; pour le verbe a anina 'accrocher, suspendre ; effleurer', nous disposons des mots dérivés : aninare 'accrochage' (cf. $L B^{e}$, s.v. Aninare), aninat 'attaché, accroché' (cf. LB', s.v. Aninatu).

Le verbe a netezi 'caresser' (cf. $L B^{e}$, s.v. Netezescu) ne présente pas de dérivés. De plus, nous devons préciser que le $L B^{e}$ enregistre des verbes qui présentent de nos jours le sème [+ toucher] dans leur description sémantique, mais qui ne le présentaient pas à l'époque. Il s'agit des verbes a mângâia 'caresser' (cf. $L B^{e}$, s.v. 
Mángảiu), dont le sens de base était 'réconforter, consoler', a dezmierda 'cajoler' (cf. $L B^{e}$, s.v. Desmerdu), ayant le sens de 'amuser, faire détendre', et a gingăși 'câliner' (cf. $L B^{e}$, s.v. Jingảsşescu), présentant toujours le seul sens d'amuser ou de faire détendre.

\section{Le micro-champ de la perception visuelle}

Organisé autour du sème [+ vue], ce micro-champ est de loin le plus large, réunissant 13 verbes, à savoir : a vedea 'voir' (cf. $L B^{e}$, s.v. Vẻdu, Vảzu, Viddu), a privi 'regarder' (cf. LB', s.v. Privescu), a (se) uita 'oublier; regarder' (cf. LB', s.v. Uitu), a (se) inholba 'fixer du regard, écarquiller les yeux' (cf. $L B^{e}$, s.v. Inholbu), a (se) involba 'écarquiller les yeux' (cf. $L B^{e}$, s.v. Involbu), a (se) zgâi 'regarder fixement, écarquiller les yeux' (cf. $L B^{e}$, s.v. Sgảescu), a stăura 'fixer du regard, regarder fixement' (cf. $L B^{e}$, s.v. Stảuru), a ochi 'viser ; apercevoir' (cf. $L B^{e}$, s.v. Ochescu), a zări 'apercevoir' (cf. $L B^{e}$, s.v. Zärescu), a spiona 'espionner' (cf. $L B^{e}$, s.v. Şpionescu), a iscodi 'épier ; espionner' (cf. $L B^{e}$, s.v. Iscodescu), a pândi 'observer attentivement, guetter' (cf. $L B^{e}$, s.v. Pảndescu), a aleşui 'guetter pour surprendre ; tendre un piège' (cf. $L B^{e}$, s.v. Aleşuescu).

L'examen de ces verbes nous permet de noter, d'un côté, que le prototype de la série est le verbe a vedea 'voir' (cf. $L B^{e}$, s.v. Vẻdu, Vảzu, Vỉdu) et, de l'autre, que la plupart d'entre eux sont en principe transitifs; néanmoins, quelques entrées présentent des valeurs pronominales ou impersonnelles (cf. $L B^{e}$, s.v. Inholbu, Involbu).

En ce qui concerne le verbe a vedea 'voir' (cf. $L B^{e}$, s.v. Vẻdu, Vảzu, Vỉdu), on note que les trois entrées représentent en fait trois manières graphiques distinctes de rendre le sens de ce verbe ; il s'agit donc d'un doublet graphique é/ỉ et d'un doublet phonétique $d / z$. L'examen de ces trois formes nous permet de saisir que les mots vedettes $v a ̉ z u$ et vỉdu sont définis par des renvois au mot védu, qui bénéficie à son tour d'une définition réalisée à l'aide des équivalents latins, hongrois et allemands, en absence d'autres éléments ou de notices informationnelles. En ce qui concerne le verbe a privi 'regarder' (cf. $L B^{e}$, s.v. Privescu), les rédacteurs placent une notice étymologique fantaisiste : l'origine latine du verbe a privi « a Lat. pervideo » est fautive - nous savons désormais que son étymologie est, en fait, slave : sl. priviti.

Quatre verbes - a (se) inholba 'fixer du regard, écarquiller les yeux' (cf. $L B^{e}$, s.v. Inholbu), a (se) învolba 'écarquiller les yeux' (cf. $L B^{e}$, s.v. Involbu), a (se) zgâi 'regarder fixement, écarquiller les yeux' (cf. $L B^{e}$, s.v. Sgảescu), a stăura 'fixer du regard, regarder fixement' (cf. $L B^{e}$, s.v. Stảuru) - appartiennent à la même série synonymique du verbe écarquiller (les yeux), dont la description sémantique inclut, hormis le sème [+ vue], le sème de modalité affective [+ stupéfaction]. En ce qui concerne leur circulation, on constate que le verbe a (se) inholba 'fixer du regard, écarquiller les yeux' (cf. $L B^{e}$, s.v. Inholbu) appartient au registre standard de la langue et qu'il a comme synonymes le verbe familier et populaire a se zgâi 'regarder fixement, écarquiller les yeux' (cf. $L B^{e}$, s.v. Sgảescu) ou le régionalisme a stăura 'fixer du regard, regarder fixement' (cf. $L B^{e}$, s.v. Stảuru). En revanche, le verbe a (se) învolba 'écarquiller les yeux' (cf. $L B^{e}$, s.v. Involbu) ne s'emploie plus aujourd'hui avec le même sens. En ce qui concerne le verbe a ochi 'viser; 
apercevoir' (cf. $L B^{e}$, s.v. Ochescu), il se trouve dans trois contextes d'emploi, alors qu'aujourd'hui on ne l'emploie qu'avec le seul sens de « viser », tandis que pour le verbe a (se) uita 'oublier ; regarder' (cf. LB', s.v. Uitu), le deuxième sens inclut les sèmes du verbe regarder. La dernière série - réunissant les verbes a spiona 'espionner' (cf. $L B^{e}$, s.v. Spionescu), a iscodi 'épier; espionner' (cf. $L B^{e}$, s.v. Iscodescu), a pândi 'observer attentivement, guetter' (cf. $L B^{e}$, s.v. Pándescu), a aleşui 'guetter pour surprendre ; tendre un piège' (cf. $L B^{e}$, s.v. Aleşuescu) présente dans sa description sémantique les sèmes [+ vue], [+ intention] et [+ attention]. Les trois premiers verbes appartiennent au registre standard de la langue (la forme littéraire du premier verbe est a spiona), tandis que l'emploi du dernier verbe est vieilli.

Il convient également de noter que presque chaque entrée présente des mots dérivés. Par exemple, pour a vedea 'voir', nous avons : vedere 'vue' (cf. $L B^{e}$, s.v. Vedére), vederos 'visible' (cf. $L B^{e}$, s.v. Vederosu), văzătoriu 'la personne qui voit ou qui regarde' (cf. $L B^{e}$, s.v. Vázảtoriu, Vẻzảtoriu) ; pour le verbe a privi 'regarder', nous trouvons : privire 'regard' (cf. LB , s.v. Privire), privitoriu 'celui qui regarde, spectateur' (cf. $L B^{e}$, s.v. Privitoriu), privitoare 'celle qui regarde, spectatrice' (cf. $L B^{e}$, s.v. Privitóre) ; pour a (se) uita 'oublier ; regarder', nous avons : uitare 'oubli ; regard' (cf. $L B^{e}$, s.v. Uitare), uitătoriu 'oublieux' (cf. $L B^{e}$, s.v. Uitảtoriu), uituc 'oublieux' (cf. $L B^{e}$, s.v. Uitucu) ; pour a înholba 'fixer du regard, écarquiller les yeux', il est donné : înholbat 'ébahi' (cf. $L B^{e}$, s.v. Inholbatu) ; pour le nom ochi 'œil' (cf. $L B^{e}$, s.v. $O c h i u$ ), on a le verbe dérivé a $o c h i$ 'viser; apercevoir' (cf. $L B^{e}$, s.v. Ochescu) et le nom dérivé ochire 'action de regarder' (cf. $L B^{e}$, s.v. Ochire); pour a iscodi 'épier ; espionner', nous avons : iscoadă 'espion, émissaire secret' (cf. $L B^{e}$, s.v. Iscódả), iscodire 'inspection, observation secrète' (cf. $L B^{e}$, s.v. Iscodire), iscoditoriu 'enquêteur' (cf. $L B^{e}$, s.v. Iscoditoriu); pour a pândi 'observer attentivement, guetter', nous lisons: pândire 'guet' (cf. $L B^{e}$, s.v. Pảndire), pânditoriu 'qqn qui observe attentivement, guetteur' (cf. $L B^{e}$, s.v. Pảnditoriu), pândariu et pândaş 'gardien, guetteur' (cf. $L B^{e}$, s.v. Pảndariu, Pảndaşu); pour a aleşui 'guetter pour surprendre ; tendre un piège', on trouve : aleşuire 'action de tendre un piège' (cf. $L B^{e}$, s.v. Aleşuire), aleşuitoriu 'celui qui guette, qui tend des pièges ; piégeur, personne rusée' (cf. $L B^{e}$, s.v. Aleşuitoriu).

Avant de passer aux conclusions, il convient de mentionner la présence de nombreuses collocations et expressions idiomatiques à repérer dans d'autres entrées, qui n'ont pourtant pas fait l'objet de notre analyse. Ainsi, le verbe a căuta 'chercher' (cf. $L B^{e}$, s.v. Cautu) peut revêtir parfois le sens de «regarder, remarquer »: «9) - cautu la quineva, queva, adeq : a) me uitu 'je regarde, je jette un regard sur qqn' : specto, adspicio, contemplor : [...]. - me cautu in oglindả 'je me contemple dans le miroir' : inspicio speculum, contemplor me in speculo : [...]. 2) lu bagu in samả 'je l'observe' : video, attendo, rationem ejus habeo : [...]. »; le verbe a prinde 'prendre, attraper' (cf. $L B^{e}$, s.v. Prèndu) peut revêtir dans certains cas le sens de « voir » ou d' « apprendre (par l'ouïe) »: «5) queva, séu pre quineva cu ochii : i. e. vỉdu, ḑảrescu 'je surprends qqc. ou qqn : i.e. je le vois' : cerno, oculis observo, oculis prehendo, Lucret. IV. 1137) [...]. » et «10) prèndu de véste 'je viens d'apprendre' : observo, animadverto : [...] »; le verbe a cădea 'tomber' (cf. $L B^{e}$, s.v. $C a d u$ ) peut avoir dans certains cas un rapport avec la vue : «17) - in ochi, i. e. mẻ 
vẻdu: vẻnu in ochi, mẻ potu vedé, privì 'sauter aux yeux, i.e. je me vois : je peux me voir, me regarder' : cado in oculos, conspicior, venio in conspectum, videor : $[\ldots] »$; le verbe a cuprinde 'contenir, saisir, embrasser' (cf. $L B^{e}$, s.v. Cuprindu) revêt plusieurs sens selon le contexte : " pre quineva in braçe, adeq : in brảçişezu 'saisir qqn dans ses bras, c'est-à-dire je l'embrasse' : comprehendo, amplector : [...], cuprèndu queva cu ochii 'je le vois, je l'examine' : visu comprehendo : [...]»; le verbe a trage 'tirer' (cf. $L B^{e}$, s.v. Tragu) et le verbe a ţipa 'jeter' (cf. $L B^{e}$, s.v. Çipu) peuvent s'associer en collocation avec le mot $œ i l:$ : 8) cu ochiul', adeq : clipescu 'cligner, faire un signe de son œil' : nicto, conniveo : [...]», «- çipu ochü spre queva 'je jette mes yeux sur qqc.' : oculos conjicio in quidpiam : [...]», etc.

\section{En guise de conclusion}

Dans la présente étude, nous nous sommes proposé de réaliser un inventaire des mots de la perception tels qu'ils sont conservés dans le $L B^{e}$. Excepté le prototype de la catégorie des verbes de la perception, a simţi 'percevoir par les sens, sentir', qui se présente sous cinq formes graphiques, et son équivalent synonymique, a pricepe 'comprendre, saisir, entendre', qui se présente sous deux formes graphiques, les données recensées (35 formes graphiques verbales) nous ont permis d'examiner plus en détail la répartition de ces vocables dans les micro-champs de la perception sensorielle. Ainsi, on a pu voir que la première place était occupée par les termes visuels (15, dont trois renvoient au même contenu, celui du verbe a vedea 'voir'; cf. supra 2.5), suivis par les termes olfactifs ( 8 ; cf. supra 2.3) et, ensuite, par les notions auditives, gustatives et tactiles (chacune d'entre elles présentant 4 mots ; cf. supra 2.1, 2.2, 2.4).

À l'intérieur de cet inventaire, on constate que seulement seize mots sont pourvus aussi d'une notice étymologique. Onze mots sont présentés par les rédacteurs avec une étymologie latine, à savoir a simţi 'sentir', lat. sentio (cf. $L B^{e}$, s.v. Simţescu, Sẻnţescu), a pricepe 'comprendre', lat. percipio (cf. $L B^{e}$, s.v. Precepu), a inţelege 'comprendre', lat. intelligo (cf. $L B^{e}$, s.v. Inţelegu), a oblici 'apprendre, entendre qqch d'inattendu', lat. obliquo (cf. $L B^{e}$, s.v. Oblicescu), a îmbuca 'goûter, manger un morceau', lat. bucca (cf. $L B^{e}$, s.v. Imbucu), a privi 'regarder', lat. pervideo (cf. $L B^{e}$, s.v. Privescu), a înholba 'fixer du regard, écarquiller les yeux', lat. in et volvo (cf. $L B^{e}$, s.v. Inholbu), a învolba 'troubler; écarquiller les yeux', lat. in et volvo (cf. $L B^{e}$, s.v. involbu), a iscodi 'épier; espionner', lat. excutio (cf. $L B^{e}$, s.v. Iscodescu), a aleşui 'guetter pour surprendre ; tendre un piège', lat. ad et lacio lexi lectum (cf. $L B^{e}$, s.v. Aleşuescu). Trois mots auraient une origine grecque : voir, ainsi, les verbes a amirosi 'sentir', gr. $\mu v \rho \imath \zeta \omega$ (cf. $L B^{e}$, s.v. Amirosu), a ulma 'flairer', gr. $\dot{\alpha} \varsigma \mu \grave{\eta}, \tilde{\eta} \varsigma, \dot{\eta}$. (cf. $L B^{e}$, s.v. Ulmu), a pipăi 'tâter, palper', gr. $\pi \circ \pi \pi v \zeta \omega$ (cf. $L B^{e}$, s.v. Pipáescu). Un mot est une création autochtone : a adurmeca 'flairer', le rou. urmá, vestigium (cf. $L B^{e}$, s.v. Adurmecu), tandis que deux mots sont des équivalents ou des emprunts à l'italien - les verbes a odora 'odorer', ital. odorare (cf. LB', s.v. Odorezu) et a ochi 'viser ; apercevoir', ital. occhieggiare (cf. $L B^{e}$, s.v. Ochescu). Un mot, enfin, est un emprunt au français : a spiona 'espionner', gall. espionner (cf. $L B^{e}$, s.v. Spionescu). 
Cinq mots présentent cependant une étymologie fautive. Il s'agit de: a desmierda 'cajoler', dont l'étymon est le lat. *dismerdare, de merda; a privi 'regarder', dont l'étymon est le slave priviti; a iscodi 'épier; espionner', dont l'étymon est le sl. ischoditi; a aleşui 'guetter pour surprendre; tendre un piège', dont l'étymon est le hongrois les, et a pipăi 'tâter, palper', dont l'étymon est le sl. pipati. De plus, la variante littéraire de l'italien occhieggiare est occhiata.

L'examen de toutes ces unités lexicales nous a permis également de constater leur remarquable productivité. Les quarante-deux formes graphiques verbales sont à la base de quatre-vingt-un dérivés. Si, dans le cas du verbe prototypique de la série de verbes de perception (a simţi), on constate la présence de sept dérivés, tandis que son synonyme (a pricepe) a produit trois dérivés, dans une hiérarchie des microchamps de la perception sensorielle, la première place est occupée par les verbes de perception visuelle ( 24 dérivés), suivis par les verbes de perception auditive (14 dérivés), olfactive (13 dérivés), gustative ( 8 dérivés) et tactile (6 dérivés). Au sein de ces micro-champs, il convient de remarquer la capacité dérivative élevée de quelques-uns; ainsi, dans le micro-champ de la perception auditive, le verbe a înţelege se trouve à l'origine de six mots, suivi par a asculta (4 mots), a auzi (3 mots) et a oblici (1 mot). Pour le micro-champ de la perception gustative on observe qu'à la base des mots dérivés se trouvent les noms gustu (5 dérivés) et bucă (3 dérivés). Pour ce qui est du micro-champ olfactif, la productivité dérivative la plus élevée revient au nom miros ( 6 dérivés), suivi par amirosu (5 dérivés), a ulma et a odora (un dérivé chacun), tandis que pour le micro-champ de la perception tactile, on constate, mis à part l'absence d'un dérivé pour a netezi, la présence de deux dérivés pour chacun des verbes suivants : a pipăi, a atinge et a anina. Dans le dernier micro-champ, celui de la perception visuelle, la première place est occupée par le verbe a pândi (5 dérivés), suivi par a vedea (4 dérivés), a privi, a uita, a iscodi et a aleşui (3 dérivés chacun), a ochi ( 2 dérivés) et a înholba (un dérivé).

En ce qui concerne le traitement lexicographique des verbes de la perception, on observe que les mots vedettes sont accompagnés dans la plupart des cas d'une information grammaticale (indiquant leur classe grammaticale et leur valeur, transitive ou neutre) ou d'une notice étymologique (16 mots). Ils ne présentent pourtant pas de définitions de nature explicative. Ils sont définis (a) soit par les contextes d'emploi accompagnés par des équivalents en latin, en allemand et en hongrois (20 mots; cf. LB' s.v. Aleşuescu, Amirosu, Aninu, Ascultu, Atingu, Gustảrescu, Gustu, Imbucảturescu, Inholbu, Inţelegu, Involbu, Mirosu, Netezescu, Ochescu, Pảndescu, Precepu, Privescu, Sgảescu, Uitu, Ulmu), (b) soit directement par des synonymes en latin, en allemand et en hongrois $\left(8\right.$ mots ; cf. $L B^{e}$, s.v. Adurmecu, Iscodescu, Oblicescu, Pipảescu, Şpionescu, Stảuru, Vẻdu, Zảrescu), (c) soit, enfin, par des renvois (10 mots; cf. $L B^{e}$, s.v. Adulmecu, Amirosescu, Mirosescu, Odorezu, Pricepu, Sẻmţiu, Sẻnţiu, Simţiu, Vảzu, Vỉdu). Il convient de souligner que dans le cas de quatre entrées verbales, les rédacteurs du $L B^{e}$ ont choisi de donner des définitions mixtes, tout en mélangeant les deux premiers critères (a) et (b) (cf. LB e, s.v. Audiu, Imbucu, Simţescu, Sẻnţescu).

Pour ce qui est de l'évolution sémantique de ces mots, le sens de base (qui a, en général, une valeur active ou transitive) est conservé jusqu'à nos jours dans presque tous les cas. Il est rare que ces mots aient perdu leur signification renvoyant à la 
perception (voir, par exemple, les sens secondaires de aninu et netezescu ou même la valeur sensorielle du verbe inholbu, cf. $L B^{e}$ ). De plus, la plupart de ces mots appartiennent au registre littéraire ou standard de la langue, seulement quelques-uns font partie du registre populaire et régional (cf. $L B^{e}$, s.v. Adurmecu, Audiu, Aud, Amirosu, Amirosescu, Aleşuescu, Gustảrescu, Imbucảturescu, Mirosescu, Oblicescu, Odorezu, Precepu, Simţescu, Sgảescu, Stảuru, Ulmu).

Quant aux aspects normatifs par rapport aux normes actuelles, il convient de remarquer tout premièrement la notation variée de certains verbes (la présence des doublets graphiques ou phonétiques) et, deuxièmement, le fait que de nombreux mots ont souffert de changements graphiques du XIX ${ }^{e}$ siècle jusqu'à nos jours. D'ailleurs, cette évolution est tout à fait compréhensible. L'écriture du roumain au $\mathrm{XIX}^{\mathrm{e}}$ siècle, qui hésite encore entre une écriture à caractères cyrilliques et une écriture étymologique à caractères latins, sera peu à peu remplacée par une orthographe phonétique à caractères latins.

\section{Annexe}

Adulmecu, care, catu. 'je flaire, flairer, flairé'. V. Adurmecu.

Adurmecu, are, atu, 'je flaire, flairer, flairé', verb. act. odoratu investigo : sagio : [...]. ex valach : urmả, vestigium.

Aleşuescu, ire, itu. 'je guette, guetter, guetté pour surprendre ; je tends, tendre, tendu (un piège)'. pảndescu : insidior, insidias tendo vel pono : [...]. a Lat. ad et lacio lexi lectum : in fraudem duco : $x$ mutata in ş more solito. Vide Orthographiam Romanam sive Latino-Valachicam Petri Májor, sub lit. x.

Aleşuire, $f$. $p l$. iri. 'action de tendre un piège' insidiatio, structio insidiarum : [...].

Aleşuitoriu, $f$. óre. $p l$. ori, $f$. óre. 'celui qui guette, qui tend des pièges ; piégeur, personne rusée' insidiator: [...].

Amirosélả, f. pl. le. 'odeur'. subst : V. Mirosu subst :

Amirosescu, ire, itu. 'je sens, sentir, senti'. V. Amirosu. verb : 'je sens'.

Amirosire, f. pl. ri. 'odorat'. Subst : mirosire : odoratio, odoratus, olfactus : [...].

Amirositoriu, $f$. óre. $p l$. ori, $f$. óre. 'odorant'. adj : verb : odorus, olens, olax : [...].

Amirositurả, f. pl. i. 'odeur'. subst : odoramen, odoramentum : [...].

Amirosu, sire, situ. 'je sens, sentir, senti'. verb. act. et neutr : 1) amirosu : p. e : cutare flóre 'je sens, par exemple, le parfum d'une fleur' : odoror, olfacio : [...]. Aquéstả flóre amiróse frumosu 'Cette fleur sent bon' : hic flos pulchre olet: [...]. 2) slobodu, dau din mène mirosu 'j'exhale une odeur' : oleo, odorem edo,

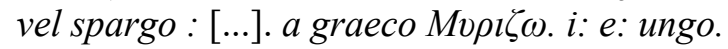

Amirosu, m. pl. uri f. 'odeur'-subst : V. Mirosu. 'odeur'.

Aninare, f. pl. ảri. 'accrochage' subst: 1) prèndere la olaltả 'lier à, attacher à' : adfixio, depensio : [...]. a Lat: lenis, $i$ : e: leniter adfigo. 2) de abiè atingere 'effleurer' : levis contactus, attactus : [...].

Aninatu, $f$. ả. $p l$. ti, $f$. te. 'attaché, accroché' inţėnatu, prensu la olaltả 'attaché, lié' : adfictus, adpensus : [...]. 2) de abiè atinsu 'effleurer' : contactus, leniter tactus, palpatus : [...].

Aninu, are, atu. ' j'accroche, accrocher, accroché ; j'effleure, effleurer, effleurer ; toucher légèrement'. verb: act: 1) inţènu, prendu la olaltả 'je lie à, j'attache à' : 
adfigo, adpendo: [...]. 2) de abiè atingu 'je touche légèrement' : contingo, leniter tango, palpo : [...].

Ascultare, $f$. $p l$. ảri ' 1 . écoute ; 2. obéissance'. subst : 1) - pentru audire 'écouter les propos d'une personne' : ascultatio, ascultatus, attentio : [...]. 2) - de porunca, dojana, séu svatul cuiva 'obéir à l'ordre ou au conseil de qqn, tenir compte de l'admonestation de qqn': ascultatio, obedientia, obtemperantia, obsequium : [...].

Ascultảtoriu, $f$. óre. $p l$. ori, f. óre ' 1 . celui qui écoute ; 2 . obéissant'. 1) subst : verb : quarele ascultả p. e. predica cuiva 'qqn qui écoute, par exemple, le sermon ou l'enseignement de qqn': ascultator, auditor: [...]. 2) adj : verb : quarele plenesce porunca, svatu, seu dojana cuiva 'obéissant': parens, obediens, obtemperans, obsequiosus, morigerus : [...].

Ascultu, are, atu 'j'écoute, écouter, écouté' verb. act : 1) - queva qua sỉ audu 'j'écoute qqc. pour entendre': Asculto, attendo, intentus sum : [...]. 2) porunca, séu svatul cuiva, adeq : primescu 'j'obéis à l'ordre ou au conseil de qqn, c'est-à-dire je le reçois' : pareo, obedio, obtempero, obsequor, morem gero : [...].

Atingere, f. pl. eri. 'toucher' subst : tactio, attactus, contactus : [...].

Atingu, gere, atinsu. 'je touche, toucher, touché'. verb: act : — queva, séu me atingu de queva 'qqc. ou je touche qqc.' : tango, attingo, contingo : [...].

Atinsu, $f$. ả. $p l$. si. $f$. se. 'touché' particip : verb : atingu. v. atingu.

Auḍire, f. pl. iri, 'ouïe', subst : auditus : [...].

Auditoriu. $f$. óre. $p l$. ori, $f$. óre. ' 1 . la personne qui écoute, 2 . ce qui peut être entendu'. I. subst : ver : auditor : [...]. II. adj : verb : audibilis, intelligibilis : $[\ldots]$.

Auditu, m. pl. uri. f. 'ouïe'. subst : V. audire.

Audiu, seu audu, vel aud, ire, itu 'j'entends, entendre, entendu'. verb : act : audio, auribus percipio : [...]. se aude 'on entend' : auditur : [...]. audu greuu 'je n'entends pas bien' : graviter, difficulter audio : [...].

Auḍu, vel aud̦. $m$. pl. uri. f. 'ouïe'. subst : auditus : [...].

Desmerdu, séu desmerdezu, are, atu. 'amuser, faire détendre ; cajoler', verb. act. I. pre quineva : 1) il desfẻtezu : oblecto, delecto : [...]. 2) vre unu pruncu, adeq : l'intrecu, lu mảdảrescu : immodicis blanditiis. et nimia indulgentia corrumpo : [...]. II. recipr. me desmerdu. 1) in desfẻtảri ne oprite : delector, oblector : [...]. 2) in resfảçare: helluor, luxurior, libidini indulgeo: [...]. 3) me intrecu: puellitor: [...]; a Lat. dis - moderor.

Gustare, f. pl. tảri. 'goûter', subst. 1) pentru ispitirea quảreiva bucate 'pour connaître un plat' : gustus, gustatus : [...]. 2) queva mảncare puçinả 'un peu de nourriture, une collation' : gustus, gustulus, gustatio, vulgo : collatio : [...].

Gustảrescu, rire, ritu. 'je goûte, goûter, goûté', verb, act. 1) gustu de multe ori 'je goûte à plusieurs reprises' : saepe gusto : [...]. 2) mảncu queva puçinu 'je goûte qqc., je mange une petite quantité de' : gusto, esito, libo : [...].

Gustosu, séu gustuosu, $f$. ósả, $p l$. oşi, $f$. óse. 'savoureux', adj. gustuosus, sapidus, boni saporis : [...]. 
Gustu, tare, tatu. 'je goûte, goûter, goûté'. verb. act. 1) vre o bucata pentru cercarea 'un plat à déguster' : gusto : [...]. 2) gustảrescu, in bucu quevá 'je goûte qqc., je mange qqc. en hâte' : libo, delibo, esito, gusto : [...].

Gustu, $m$. pl, turi, $f$. 'goût, saveur', subst. 1) $V$. Gustare. 2) - quarele éste in queva bucatả 'qui se trouve dans ce plat' : gustus, sapor : [...]. 3) V. Apetitu.

Imbucảturả, f. pl. i. 'morceau', subst. buccea, bolus, offa, frustum : [...].

Imbucảturescu, ire. itu, 'je mange, mange, mangé ; je goûte, goûter, goûté'. I. activ. pre quineva 'qqn', 1) ẻi dau quỏte o imbucảturả 'je lui donne un morceau' : offatim cibo: $[\ldots]$, 2) i. e. ẻi dau se mảnce 'je lui donne à manger' : cibo, adesco : [...]. II. neutr. i. e. gustarescu 'je goûte qqc.' : $V$. Imbucu Nro. 2) 'je mange qqc. en hâte ; je goûte qqc.'.

Imbucảturuţa, f.pl. e. 'un petit morceau', offala, offella, buccea : [...].

Imbucu, are, atu. 'je mange, mange, mangé ; je goûte, goûter, goûté', verb. act. 1) sublabro, mordeo, in buccam indo: [...]; 2) i. e. gustảrescu 'je goûte, goûter, goûté' : libo, gusto, esito : [...]; a Lat. bucca.

Inholbatu, $f$. tả, pl. m. i.f. e. 'ébahi' adj. magnos et deformes oculos habens : [...].

Inholbu, bare, atu. 'fixer du regard ; écarquiller les yeux', mẻ inholbu 'j'écarquille les yeux', verb. recip. a) mẻ uitu la queva cu ỉncremenire 'je regarde qqc. avec étonnement ou avec stupéfaction' : cum stupore intueor : [...]. b) $i$. e. intorcu ochii 'je tourne les yeux' : oculos contorqueo : [...]. c) cascu gura la queva 'je regarde qqc. bouche-bée' : hianti ore contemplor: [...]. a Lat. in et volvo, v. mutato in $h$. vide cit. Orthogr.

Intelegere, $f . p l$, i. 'compréhension, entendement'. 1) subst. verb. verbi inţelégu 'je comprends': intellectio: [...]. 2) i. e. putére de a inţelége 'la capacité à comprendre' : intellectus, intelligentia, mens : [...]. 3) $i$. e. nẻravélả : consensus, consentio, concordia: [...]. 4) a quảruiva d̦isả séu cuvẻntu, i. e. insẻmnare 'comprendre les propos d'une personne' : sensus, vis, significatio: [...]. a Lat. intelligentia : Ital. intelligenza.

Inţelegetoriu, tóre, $p l . \mathrm{i}, f$. e. 'compréhensif' adj. Intelligens : [...]. Ital. intelligente. Inţelegu, gere, lesu 'je comprends, comprendre, compris'. I. verb. act. 1) queva i.e. pricepu, cuprèndu cu mèntea 'je comprends qqc.' : intelligo, vel juxta Plaut. in Bachid. intellego, percipio, perspicio, assequor : [...]. 2) i. e. audu, scirescu, oblicescu 'j'entends, je sais, je saisis' : disco, comperio, rescio, inaudio, certior fio : [...]. II. reciproc. mẻ inţelegu cu quineva 'je conviens avec qqn sur qqc.' : a) despre queva i. e. facu nảrẻvélả, aşed̦emẻntu 'je conviens avec qqn sur qqc.' : condico, convenio, consentio : [...]. 2) i. e. petrecu cu quineva in inţelégere, in nerảvélả 'vivre en paix avec qqn, s'amuser avec qqn' : convenio, consentio cum quopiam, convenit inter nos : [...]. a Lat. intelligo.

Involbu, bare, atu. 'je me trouble, se troubler, troublé ; j'écarquille, écarquiller les yeux, écarquillé' verb. act. 1) i. e. turburu, prequum p.e. vẻntulu invólbả norii 'je me trouble ; je m'agite tout comme, par exemple, le vent agite les nuages' : perturbo, conturbo: [...]. 2) deponens. mẻ involbu, i. e. mẻ inbolbu 'j'écarquille les yeux'. $V$. Inholbu. a Lat. in et volvo.

Iscódả, f.pl. e. 'espion, émissaire secret' subst. explorator, speculator, otacusta, vel otacustes : [...], spion 'espion' : [...]. a Graeco $\omega \tau \alpha \kappa o v \varsigma \tau \varepsilon \omega$, auribus capto, quae dicuntur. 
Iscodescu, dire, itu. 'j'épie, épier, épié ; recueillir des informations', verb. act. exploro, speculor: [...]. a Lat. excutio.

Iscodire, $f . p l$. i. 'inspection, observation secrète' subst. speculatio, investigatio, exploratio : [...].

Iscoditoriu, $m . p l$. i. 'enquêteur' subst. $V$. Iscódả.

Jingảşescu, şire, itu. 'amuser, faire détendre ; câliner'. I. activ. 1) pre quineva, p.e. vre unu pruncu, i. e. desmerdu : nimis indulgenter habeo, placide admodum tracto: [...]. 2) in queva, $p$. e. in mảncảri, $i$. e. facu multả alégere : nimium selectum adhibeo : [...].

Mảngảiu, iére, étu. 'réconforter, consoler ; caresser', verb. act. solor, consolor, recreo, solatium adfero, do, praebeo, admoveo, solatio sum : [...].

Mirosélả, $f . p l$. le. 'odeur', $V$. Mirosu subst.

Mirosescu, sire, itu. 'je sens, sentir, senti', $V$. Mirosu verb.

Mirosire, f. pl. ri. 'odorat', subst. odoratio, odoratus, olfactus : [...].

Mirositurả, $f . p l$. ri. 'odeur', subst. odoramen, odoramentum, odor suavis : [...]. Grae. $\eta \delta$ vo $\mu l \alpha$ ador gratus.

Mirositoriu, $f$. tóre, $p l$. tori, $f$. tóre. 'odorant', adj. verbale : odorus, olens, olax : [...].

Mirosu, sire, itu. 'je sens, sentir, senti', verb. act. 1) queva : adeq : amirosescu $p$. e. vreo flóre 'qqc., c'est-à-dire je sens, par exemple, le parfum d'une fleur' : odoror, olfacio : [...]. 2) adeq. slobodu din mène vre unu mirosu 'j' exhale une odeur' : oleo, odorem edo, vel spargo : [...]. — de éste mirosul' plảcutu 'si cette odeur est agréable' : bene oleo, svaviter oleo : [...].

Mirosu, $m . p l$. uri 'odeur, odorat', subst. 1) una dintre quéle quinque senţiri a le omului 'l'un des cinq sens du corps humain'; odoratus, olfactus : [...]. - 2) quarele ése din vreo flóre 'qui sort d'une fleur' : odor : [...]. - mirosu bunu, formosu, placutu 'une bonne ou une belle odeur, une odeur agréable': odor gratus, svavis : [...]. - mirosu greu 'une odeur lourde' : odor gravis, ingratus, graveolentia: [...]. Grae. $\mu v \rho \omega \delta \imath \alpha$ odor, olfactus.

Ne ascultare, $f . p l$. ảri 'désobéissance'. subst. inobedientia : [...].

$\mathrm{Ne}$ ascultảtoriu, $f$. tóre, $p l$. ri, $f$. re 'désobéissant'. adj. inobedus, inobediens, haud obtemporans : [...]. i. e. qui non auscultat.

Ne audzitu, $f$. tả, $p l$. ţi, $f$. te 'inouï' adj. inauditus : [...].

Negustatu, $f$. tả, $p l$. ţi, $f$. te. 'que l'on n'a pas goûté', adj. ingustatus : [...].

$\mathrm{Ne}$ ỉnţelégere, $f$. 'incompréhension'. subst. 1) p. e. a quảruiva cuvẻntu 'd'un mot' : obscuritas : [...]. - 2) i. e. nepricépere 'ignorance' : imperitia, imprudentia, inscitia : [...]. - 3) $V$. Ne ascultare 'désobéissance'. - 4) intrả doi, séu mai mulţi la olaltả 'entre deux ou plusieurs' : dissensio, dissidium, discordia : [...].

$\mathrm{Ne}$ inţeleghẻtoriu, $f$. tóre, $p l$. ri, $f$. óre. 'incompréhensif'. adj. 1) i. e. nepriceputu 'ignorant' : imperitus, inscius, imprudens : [...]. - 2) V. Ne ascultảtoriu 'désobéissant'.

Ne inţelesu, $m$. pl. uri, $f$. 'obscurité'. subst. $V$. Ne inţelégere.

Ne inţelesu, $f$. lésả, $p l$. ši, $f$. se. 'incompris, confus', adj. 1) que nu se póte ințtelége 'qui ne peut être compris, obscur' : obscurus, non perspicuus : [...]. - 2) $V . \mathrm{Ne}$ inţeleghẻtoriu. - 3) $V$. Ne ascultảtoriu.

Nemirositoriu, $f$. tóre, $p l$. ri, $f$. re. 'inodore'. adj. inodorus, inolens : [...].

Nepipảitu, $f$. tả, $p l$. ţi, $f$. te. 'impalpable' adj. intactus, haud contrectatus : [...]. 
Nesẻmţire, $f$. $p l$. ri. 'insensibilité ; défaillance, évanouissement'. subst. inopia sensus : [...].

Nesẻmţitoriu, $f$. tóre, $p l$. ri, $f$. re. 'insensible'. adj. expers sensus, insensatus : [...].

Nesimţire, 'insensibilité ; défaillance, évanouissement'. $V$. Nesẻmţire.

Netezescu, ire, itu. 'je je lisse, lisser, lissé ; toucher, caresser', verb. act. 1) i. e. facu néted, p.e. vre o ţeseturả 'je lisse, par exemple, une étoffe' etc. laevigo, glabro : [...]. 2) pre quineva, vre o mỉçiả, vre un puiu, caţelu 'je caresse qqn ou qqc., par exemple un chat, un animal, un chiot' etc. mulceo, palpor : [...].

Oblicescu, cire, itu 'j'apprends, j'entends qqc. d'inattendu'. verb. act. disco, comperio, rescio, inaudio : [...]. a Lat. obliquo, quasi via obliqua, indirecta aliquid comperio.

Oblicire 'entendement', f. pl. ri. subst. auditio : [...].

Ochescu, chire, itu. 'je vise, viser, visé ; j'aperçois, apercevoir, aperçu'. verb. act. 1) i. e. aruncu ochii spre queva 'je jette un regard, un coup d'œil sur qqc.' : oculos, conjicio, vel adjicio ad aliquid: [...]. - 2) i. e. cautu, séu mẻ uitu la queva 'je cherche du regard ou je regarde qqc.' : inspicio, contemplor quidpiam : [...]. - 3) i. e. clipescu, facu, séu tragu cu ochiul' 'je cligne des yeux, je fais un signe à qqn en clignant de l'œil' : oculis blandior: [...]. Ital. occhieggiare. nictare, amicis oculis videre.

Ochire, f. pl. ri. 'action de regarder' subst. inspectio, contemplatio : [...].

Odorélả, $f . p l$. le. 'odeur'. $V$. Mirosu 'odeur'.

Odorezu, ire, itu. 'j'odore, odorer, odoré'. verb. act. V. Mirosu 'je sens'. Ital. odorare. olfacere.

Pảndariu, $m . p l$. ri. Pảndaşu, $m$. pl. şi. 'gardien, guetteur' subst. custos : [...].p. e. la vie 'dans le vignoble' : custos vineae : [...].

Pảndescu, dire, itu. 'j'observe, observer, observé attentivement ; je guette, guetter, guetté'. verb. act. 1) p. e. viea, pảduré, ţérina, etc. i. e. pảzescu 'le vignoble, la forêt, une terre etc. i.e. je surveille' : custodio : [...]. - 2) dupẻ queva, séu quineva 'qqc. ou qq' : a) $i$. e. aşteptu cu luare de samả 'j'attends en alerte' : expecto, praestolor : [...]. b) pre quineva, i. e. iéu sama dupẻ quineva 'qqn, i.e. je l'observe' : servo, observo, aucupor, speculor: [...].

Pảndire, f. pl. ri. 'guet' subst. 1) i. e. pazả 'garde' : custodia : [...]. 2) speculatio : $[\ldots]$.

Pảnditoriu, $m$. pl. ri. 'qqn qui observe attentivement, guetteur' subst. 1) V. Pảndaşu. -2) speculator, insidiator: [...].

Pipảescu, ire, itu. 'je palpe, palper, palpé'. verb. act. palpo, adtrecto, contrecto : [...]. Graecis $\pi о \pi \pi \nu \zeta \omega$ i. e. leniter contrecto.

Pipảire, f. pl. ri. 'toucher; attouchement' subst. palpatio, adtrectatio, contrectatio : $[\ldots]$.

Precepảtoriu, $f$. tóre, $p l$. ri, $f$. re. 'savant', adj. inţelegẻtoriu : intelligens, perspicax, perspiciens, prudens, peritus : [...].

Precépere, $f . p l$. ri. 'compréhension, intelligence, entendement', subst. perceptio, intellectus, intelligentia, prudentia, perspicacia, peritia : [...].

Preceputu, $f$. tả, $p l$. ţi, $f$. te. 'compétent, habile', subst. 1) partitip. verbi : precep' 'je comprends' $: V$. Precepu. 2) $V$. Pecepẻtoriu. 
Precepu, cépere, put. 'je comprends, comprendre, compris'. verb. act. 1) i. e. inţelegu 'je comprends' : intelligo, percipio : [...]. p. e. precepi tu lảtinesce ? 'comprends-tu le latin ?' intelligisne latine ? [...]. - 2) i. e. prind' de véste 'apprendre qqc, connaître par une information', bagu de samả 'j'observe, je me rends compte de qqc.' : observo, animadverto, sentio, perspicio, deprehendo : [...]. p. e. bẻne precepu io cảnia ta 'je me rends compte bien de ta méchanceté' : probe observo (novi) tuam astutiam : [...]. - 3) queva sciènţả, séu mảiestrie, $i$. e. sciu 'qq. science ou art, i.e. je sais' : calleo, novi, scio : [...].p. e. bène precépe rẻndul' èconomii, gảzdảsagul 'l'administration connaît bien l'affaire de l'économie' : rite scit, callet oeconomiam : [...]. a Lat. percipio.

Pricepu, ere, utu. 'je comprends, comprendre, compris'. et deriv. v. act. V. Precepu.

Privescu, vire, vitu. 'je regarde, regarder, regardé'. verb. act. cautu, mẻ uitu 'je cherche du regard, je regarde' : specto, adspicio, intueor, cerno, contemplor : [...]. a Lat. pervideo.

Privire, f. pl. ri. 'regard' subst. spectatio, adspectus, intuitus, contemplatio : [...].

Privitoriu, $m . p l$. ri. 'celui qui regarde, spectateur' subst. cảutảtoriu 'celui qui observe' : spectator: [...]. — Privitóre 'celle qui regarde, spectatrice', spectatrix : [...].

Sẻmţiu, ţire, ţitu 'je sens, sentir, senti'. deriv : $V$. sỉmţiu.

Sẻnţescu, séu : sỉmţescu, ţire, ţitu 'je sens, sentir, senti'. verb. activ. 1) sentio : [...]. 2) i. e. prèndu de véste 'apprendre qqc., connaître par une information': observo ; animadverto, sentio : [...]. a Lat. sentio. Ital. sentire.

Sẻnţire, séu : sỉmţire, f. pl. ri. 'sens'. subst. Sensus : [...].

Sẻnţiu, ţire, ţitu. Sẻntu, ţire, titu. 'je sens, sentir, senti'. verb. activ. V. Sẻnţescu.

Sẻnţitoriu, $m$. pl. ri. 'la personne sensible'. subst. sentiens, plenus sensu : [...].

Sgảescu, ire, itu. 'je regarde, regarder, regardé ; j'écarquille les yeux, écarquiller les yeux, écarquillé'. verb. act. mẻ sgảescu, i. e. mẻ ỉnvolbu 'je regarde fixement, i.e. j'écarquille les yeux' : cum stupore intueor : [...].

Simţire, f. pl. ri. 'sens'. subst. Sensus : [...].

Simţitoriu, $f$. tóre, $p l$. ri, $f$. re. 'sensible'. adj. sentiens, plenus sensu : [...].

Simţescu, ţire, ţitu 'je sens, sentir, senti'. verb. act. 1) entio : [...]. 2) i. e. prèndu de véste 'apprendre qqc., connaître par une information' : observo, animadverto, sentio : [...]. a Lat. sentio.

Simţiu, țire, ţitu. Simtu, ţire, ţitu. 'je sens, sentir, senti'. verb. act. V. Simţescu.

Şpionescu, nire, nitu. 'j'épie, épier, espionner, épié, espionné'. verb. act. exploro, speculor : [...]. Gall. espionner.

Şpionu, $m . p l$. ni. 'espion' subst. adeq: iscódả 'espion, émissaire secret' : explorator, speculator : [...]. Gall. espion.

Stảuru, rare, ratu. 'je fixe du regard, fixer du regard, (avoir le regard) fixé'. verb. activ. diu specto, cum stupore intueor : [...].

Uitare. 'oubli ; regard' f. subst. 1) oblivio, oblivium : [...]. 2) i. e. privire, cảutare 'regard' : spectatio, adspectus, intuitus, contemplatio : [...]. a Lat. intuitus, per Metathesim.

Uitảtoriu, $f$. tóre, $p l$. ri, f. re. 'oublieux' adj. obliviosus, obliviscens : [...].

Uitu, tare, tatu. 'j'oublie, oublier, oublié ; je regarde, regarder, regardé'. I. verb. act. queva, séu imi uitu de queva 'qqc. ou j'oublie qqc.' : obliviscor, oblivioni do : 
[...]. II. mẻ uitu 'je regarde' : 1) la quineva, séu queva, i. e. cautu, privescu 'qqn ou qqc., i.e. je cherche du regard, je regarde' : specto, adspicio, intueor, contemplor, cerno : [...]. 2) pre quineva, séu pre queva, i. e. îl' socotescu, îl' bagu în samả 'qqn ou qqc., i.e. 'je remarque qqn, j'ai de la considération pour lui' : attendo, respicio, rationem habeo: [...]. p. e. nu se uitả néque pre o poruncả 'il n'obéit à aucun ordre, il n'a pas de considération pour les ordres' : nulla jussa respicit : [...].

Uitucu, tucả. 'oublieux' $V$. Uitảtoriu.

Ulmare. f. 'flairée'. subst. odoratus, olfactus [...].

Ulmu, mare, matu. 'je flaire, flairer, flairé'. verb. act. 1) despre omu, i. e. amirosescu 'à propos de l'homme, i. e. je flaire, je perçois l'odeur de qqc.' : olfacio, odoror : [...]. 2) despre cảne 'à propos d'un chien' : sagio : [...]. a Graec. $\dot{\delta} \varsigma \mu \grave{\eta}, \tilde{\eta} \varsigma, \dot{\eta}$. odor.

Vảzảtoriu, $m . p l$. ri. 'la personne qui voit ou qui regarde' subst. $V$. Vẻzẻtoriu.

Vảzu, vedére, zutu. 'je vois, voir, vu'. $V$. Vẻdu.

Vedére, f. pl. ri. 'vue' subst. 1) visio, visus, conspectus : [...]. — simţiré vederii 'le sens de la vue' : [...]. 2) adeq. vedenie, séu lucru, que se véde 'vision ou chose que l'on voit' : spectaculum, facies, visio, adspectus, conspectus : [...].

Vederosu, $f$. rósả, $p l$. şi, $f$. se. 'visible' adj. 1) que se póte bène vedé 'ce que l'on peut voir bien' : visibilis, adspectabilis, conspicuus : [...]. 2) unde se véde bène, p. e. vreo casả 'où l'on peut voir bien, par exemple dans une maison : luminosus, lucidus : [...].

Vẻdu, dére, dutu. 'je vois, voir, vu'. verb. act. video, cerno, oculis usurpo : [...].

Vẻzảtoriu, $m$. $p l$. óre, $p l$. ri, $f$. óre. 'la personne qui voit ou qui regarde' adj. visor, videns : [...].

Vỉdu, dere, dutu. 'je vois, voir, vu'. $V$. Vẻdu.

Zảrescu, rire, ritu. 'j'aperçois, apercevoir, aperçu'. verb. act. cerno, specto, oculis observo: [...].» (cf. $L B^{e}, \quad$ repéré à: <http://dspace.bcucluj.ro/jspui/handle/123456789/48723)>

\section{BIBLIOGRAPHIE}

ALDEA Maria (2007), Dicționarul - instrument de recunoaștere culturală. Studiu de caz: Lexiconul de la Buda (1825), in : DiNDELEGAN Gabriela Pană (éd), Limba română - stadiul actual al cercetării. Actele celui de al 6-lea Colocviu al Catedrei de Limba Română, 29-30 noiembrie 2006, București, Editura Universităţii din București, p. 651-654.

ALDEA Maria (2012), Câteva observații asupra discursului lexicografic din secolul al XIX-lea, in : PALENíKOVÁ Jana, SITAR-TAUT Daniela (éd.), Zilele studiilor romanice (II). Teorii, modele noi și aplicarea lor în lingvistica, literatura, translatologia și didactica ultimilor 20 de ani, Bratislava, septembrie 2011, Bratislava, Univerzita Komenského, p. 102-110.

AlDEA Maria (2013), Avant-propos, $L B^{e}$. Disponible sur : $<\mathrm{http} / / /$ dspace.bcucluj.ro/jspui/handle/123456789/48723>.

Dima Gabriela (2002), Verbele sentiendi în limbile engleză şi română, Galaţi, Editura Fundaţiei Universitare « Dunărea de Jos ». 
MunteAnU Romul (1997), Școala Ardeleană : studii, București, Casa Editorială Odeon.

NiCULA Irina (2012), Modalităţi de exprimare a percepțiilor fizice. Verbele de perceptie în limba română, București, Editura Universităţii din București.

$D L R^{a}=$ Dicționarul limbii române, Ediţie anastatică după Dicţionarul limbii române (DA) şi Dicţionarul limbii române (DLR), București, Editura Academiei Române, 2010.

$L B^{e}=$ Aldea, M. (sous la direction de) (2013). Lesicon românescu-latinescuungurescu-nemţescu [...]. Seu Lexicon valachico-latino-hungaricogermanicum [...], Budae, Typis et Sumtibus Typografiae Regiae Universitatis Hungaricae, 1825. Édition électronique de Maria Aldea, Daniel Corneliu Leucuța, Lilla Marta Vremir, Vasilica Eugenia Cristea, Adrian Aurel Podaru. Cluj-Napoca. Disponible sur :

$<$ http://dspace.bcucluj.ro/jspui/handle/123456789/48723>. 